\title{
An Average Equator for the Geomagnetic $\mathrm{S}_{q}$-Field*
}

\author{
By Hiroshi MAEDA \\ (Geophysical Institute, Kyoty University)
}

\begin{abstract}
In the preceding paper [1], we obtained a coordinate system for the geomagnetic $\mathrm{S}_{q}$-field. The equator of this spherical coordinate may be regarded as an average equator for the $S_{7}$-field.

This equator is in good agreement with a line of eastward maximum current density calculated by the dynamo-theory taking account of the discrepancy between the geomagnetic and geographic axes and of the high electric conductivity in the equatorial zone.

These results seem to suggest the fairly deep-rooted effect of the dynamo-action due to the discrepancy of above two axes upon the $S_{q}$-field on one hand, and the high electric conductivity in the equatorial zone on the other.
\end{abstract}

\section{Introduction}

Since an anomalous daily variation of horizontal intensity at Huancayo had been pointed out by H.E. Johnston and A.G. McNish [2], this interesting problem of diurnal variation of geomagnetism in equatorial regions has been discussed by many workers. M. Hasegawa has for a long time entertained the idea that these anomalous regions encircle the Earth, and he, with M. Ota, estimated such a line (say $\boldsymbol{M S}$ equator) from an analysis of the data for the Polar Year, 1932-33 [3]. Recently A.T. Price and G.A. Wilkins [4] have also estimated a line of maximum $\Delta H$. These two lines are in good agreement within the limits of error (Fig. 1). As a result of tem. porary observations in Africa, India and America, J. Egedal [5] has pointed out that these anomalous regions are situated along the magnetic equator. But from a result of temporary observations in south India and Ceylon by B.L. Gulatee [6], and by S.K. Pramanik and S.Y. Narayanan [7], it should seem that the line of maximum $\Delta H$ does not lie closely on the magnetic equator.

If $S_{q}$ is due to an electric current flowing in the so-called $S_{q}$-layer, there must be expected to be an anomalous concentration of electric current (say electrojet by S. Chapman [8]) in these regions. On the origin of an equatorial electrojet, A.G. McNish [9] attributed it to an effect of the discrepancy between the magnetic and geographic equators upon the dynamo-action in the $S_{7}$-layer, and D.F. Martyn [10] to an increase of the electric conductivity near the magnetic equator. Recently M. Hirono [11], K. Maeda [12], T.G. Cowling [13], D.F. Martyn and W.G. Baker [14] have discussed,

* Contribution of Geophysical Papers dedicated to Prof. M. Hasegawa on his sixtieth birthday. 
independently, the conductivity of the ionosphere from a same point of view (Hall polarization mechanism), and given an explanation for the enhanced conductivity near the magnetic equator.

We find here a line of eastward maximum current density at $11 \mathrm{~h} 16 \mathrm{~m}$ local time, at which $H$ is nearly maximum, on the basis of the dynamo-theory, taking account of the discrepancy between the geomagnetic and geographic axes and of high conductivity in the equatorial zone, and compared with the equator of our coordinate system for the $\mathrm{S}_{q}$-field.

\section{An Average equator for the $S_{q}$-field}

The equator of our coordinate system obtained in the preceding paper [1] may be

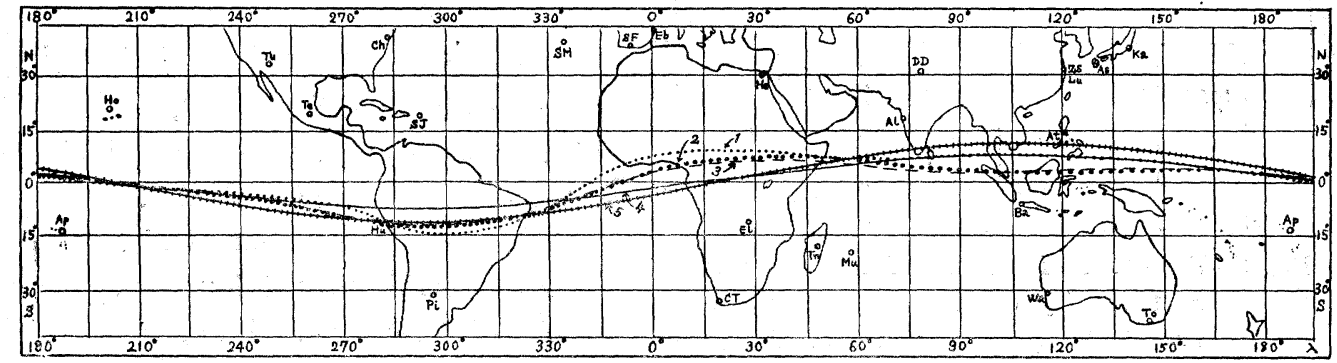

Fig. 1-Equator of our coordinate system as compared with several equators, where $1=$ magnetic equator, $2=$ line of maximum $\Delta H$ (After A.T. Price and G.A. Wilkins), $3=$ line of maximum $a_{1}$ (After M. Hasegawa and M. Ota), $4=$ equator of our coordinate system, $5=$ geomagnetic equator.

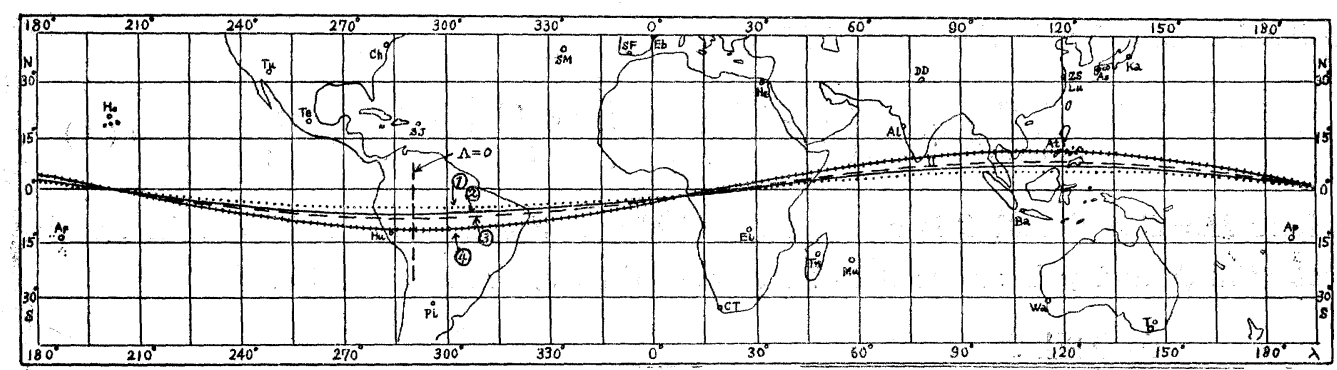

Fig. 2-Lines of eastward maximum current density at $11 \mathrm{~h} 16 \mathrm{~m}$ local time in the cases of $f=1$ (1), $f=5$ (2), and $f=100$ (3), as compared with the geomagnetic eqnator (4).

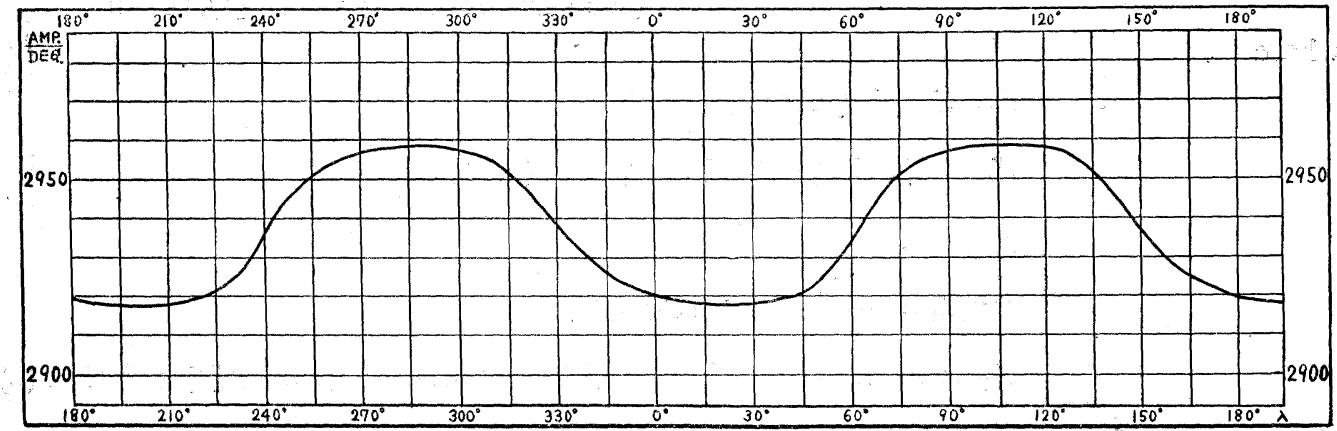

Fig. 3-Variation of current density along the line of its maximum in the case of $f=5$, 


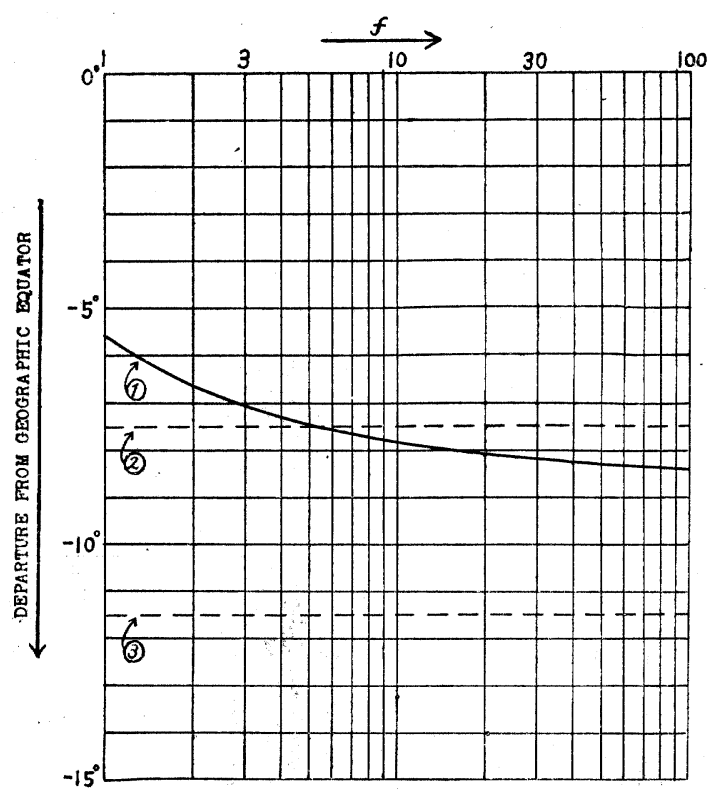

Fig. 5-Departure of the line of maximum current density (1), of the equator of our coordinate system (2) and of the geomagnetic equator (3) from the geographic equator on the meridian of $\Lambda=0$. regarded as an average equator for $S_{q}$, and is illustrated in Fig. 1 as compared with other several equators. This average equator for $S_{q}$ is in good agreement with that derived from the dynamotheory as below.

The notation used is as follows: $\theta, \lambda=$ geographic colatitude and east longitude, $\tau=$ geomagnetic local time,

$\Theta, A=$ geomagnetic colatitude and east longitude,

$T=$ geomagnetic universal time (i.e. $-69^{\circ}$ meridian time),

$U, V=$ southward and eastward components of air velocity, $\phi=$ velocity potential of air, $R=$ electric current function, $K=$ electric conductivity, $H_{z}=$ vertical component of the earth's magnetic field, $a=$ radius of the $S_{q}$-layer.

It is well known that the current function $R$ for the stationary state must satisfy the following equation in the case of $K=$ const. :

$$
\frac{\partial^{2} R}{\sin \Theta \partial \Lambda^{2}}+\frac{\partial}{\partial \Theta}\left(\sin \Theta \frac{\partial R}{\partial \Theta}\right)=a K\left\{\frac{\partial\left(V H_{z}\right)}{\partial \Lambda}+\frac{\partial\left(U H_{z} \sin \Theta\right)}{\partial \Theta}\right\}
$$

where $H_{z}=C \cos \Theta$ and $C$ is a constant. If $U$ and $V$ can be derived from the velocity potential $\psi$, and $\psi$ is assumed to be expressed by surface harmonics.

$$
\begin{aligned}
& \psi=\sum_{n}^{n} \sum_{n}^{m} \psi_{n}^{m} \\
&=\sum_{n}^{m} \sum_{n}^{m} k_{n}^{m} P_{n}^{m}(\cos \theta) \sin \left(m \tau+\alpha_{n}^{m}\right) \quad \ldots \ldots \text { in geographic coordinate, } \\
&=\sum_{n}^{n} \sum_{m} k_{n}^{m} \sum_{\mu} c_{m}^{\mu} P_{n}^{\mu}(\cos \Theta) \sin \left(\mu \Lambda+\beta_{n}^{\mu}\right) \ldots \ldots \text { in geomagnetic coordinate, } \\
& \text { (see our preceding paper [1], p. 48) }
\end{aligned}
$$

where

$$
\begin{aligned}
& c_{m}^{0}=A_{0 m} \sin \left(m T+\alpha_{n}^{m}\right), \\
& c_{m}^{\mu}=\left\{A_{\mu m}^{2} \sin ^{2}\left(m T+\alpha_{n}^{m}\right)+B_{\mu m}^{2} \cos ^{2}\left(m T+\alpha_{n}^{m}\right)\right\}^{\frac{1}{2}}, \\
& \beta_{m}^{0}=\frac{\pi}{2}, \\
& \tan \beta_{m}^{\mu}=-\frac{A_{\mu_{m}}}{B_{\mu_{m}}} \tan \left(m T+\alpha_{n}^{m}\right), \\
& A_{\mu_{m}} \text { and } B_{\mu_{m}}=\text { notations used in our preceding paper [1]. relation (3). }
\end{aligned}
$$

then the solution of eq. (1) is 


$$
R=-K C \sum_{n} \sum_{m} k_{n}^{m} \sum_{\mu}\left[c_{m}^{\mu} S_{n}^{\mu}+C_{I}^{\mu} p^{\mu}+C_{I I}^{\mu} q^{\mu}\right] \sin \left(\mu \Lambda+\beta_{m}^{\mu}\right),
$$

where

$$
\begin{aligned}
& S_{n}^{\mu}=\frac{n\left[(n+1)^{2}-\mu^{2}\right]^{\frac{1}{2}}}{(n+1)(2 n+1)} P_{n+1}^{\mu}+\frac{(n+1)\left[n^{2}+\mu^{2}\right]^{\frac{1}{2}}}{n(2 n+1)} P_{n-1}^{\mu}, \\
& p^{\mu}=\frac{\sin ^{\mu} \Theta}{(1+\cos \Theta)^{\mu}}, \quad q^{\mu}=\frac{\sin ^{\mu} \Theta}{(1-\cos \Theta)^{\mu}}, \\
& C_{I}^{\mu}, C_{I I}^{\mu}=\text { arbitrary constants. }
\end{aligned}
$$

Assuming that the electric conductivity of the layer concerned is $f K_{0}$ for region II and $K_{0}$ for regions I and III (Fig. 4), the solution for these three regions is

$$
\left.\begin{array}{r}
R_{I}=-K_{0} C \sum_{n} \sum_{m} k_{n}^{m} \sum_{\mu}\left[c_{m}^{\mu} S_{n}^{\mu}+C_{I}^{\mu} p^{\mu}\right] \sin (\mu \Lambda \\
\left.+\beta_{m}^{\mu}\right), \\
R_{I I}=-f K_{0} C \sum_{n} \sum_{m} k_{n}^{m} \sum_{\mu}\left[c_{m}^{\mu} S_{n}^{\mu}+C_{I I}^{\mu} p^{\mu}+C_{I I I}^{\mu} q^{\mu}\right] \\
\sin \left(\mu \Lambda+\beta_{m}^{\mu}\right), \\
R_{I I I}=-K_{0} C \sum_{n} \sum_{m} k_{n}^{m} \sum_{\mu}\left[c_{m}^{\mu} S_{n}^{\mu}+C_{I V}^{\mu} q^{\mu}\right] \sin (\mu \Lambda \\
\left.+\beta_{m}^{\mu}\right),
\end{array}\right\}
$$

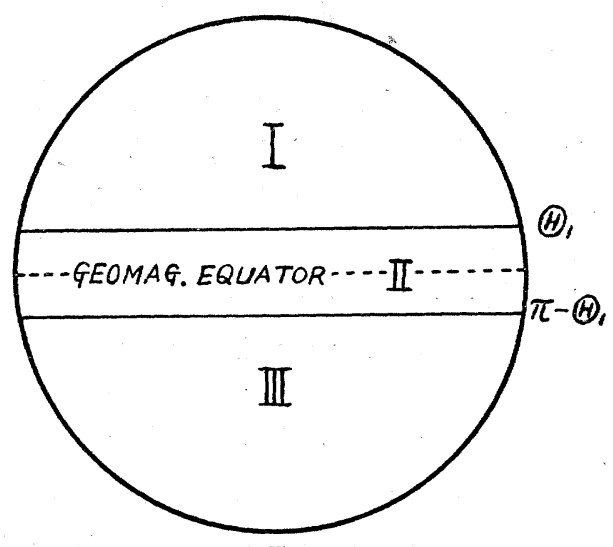

and using the condition that the normal

Fig. 4

component of the current and the tangential component of the electric field are continuous at the two boundaries of the three regions, the arbitrary constants are determined as follows:

$$
C_{I}^{\mu}=\frac{\Delta_{I}^{\mu}}{\Delta^{\mu}}, \quad C_{I I}^{\mu}=\frac{\Delta_{I I}^{\mu}}{\Delta^{\mu}}, \quad C_{I I I}^{\mu}=\frac{\Delta_{I I I}^{\mu}}{\Delta^{\mu}}, \quad C_{I V}^{\mu}=\frac{\Delta_{I V}^{\mu}}{\Delta^{\mu}},
$$

where

$$
\begin{aligned}
& \Delta^{\mu}=(f-1)^{2}-M^{2 \mu}(f+1)^{2}, \\
& \Delta_{I}^{\mu}=(f-1) c_{m}^{\mu} W_{n}^{\mu}\left[(-1)^{\mu+m+1} 2 f M^{\mu}-(f-1)-M^{2 \mu}(f+1)\right], \\
& \Delta_{I I}^{\mu}=(f-1) c_{m}^{\mu} W_{m}^{\mu}\left[(-1)^{\mu+m+1} M^{\mu}(f+1)-(f-1)\right], \\
& \Delta_{I I I}^{\mu}=(f-1) c_{m}^{\mu} W_{n}^{\mu}\left[M^{\mu}(f+1)-(-1)^{\mu+m+1}(f-1)\right], \\
& \Delta_{T V}^{\mu}=(f-1) c_{m}^{\mu} W_{n}^{\mu}\left[2 f M^{\mu}-(-1)^{\mu+m+1}\left\{(f-1)+M^{2 \mu}(f+1)\right\}\right], \\
& \qquad M^{\mu}=\left(\frac{1+\cos \Theta_{1}}{1-\cos \Theta_{1}}\right)^{\mu}, \quad W_{n}^{\mu}=\frac{S_{n}^{\mu}\left(\Theta_{1}\right)}{p^{\mu}\left(\Theta_{1}\right)} .
\end{aligned}
$$

Table 1

$V_{e}=r_{0} \sum_{n} \sum_{m} E_{n}^{m} P_{n}^{m} \sin \left(m t+e_{n}^{m}\right)$

\begin{tabular}{|cc|c|c|}
\hline$m$ & $n$ & $E_{n}^{m}$ & $e_{n}^{m}$ \\
\hline 1 & 2 & $7.22 \gamma$ & $101^{\circ}$ \\
2 & 3 & $3.70 \gamma$ & $105^{\circ}$ \\
3 & 4 & $1.10 \gamma$ & $125^{\circ}$ \\
\hline
\end{tabular}

(After M, Hasegawa and M, Ota)
From the assumption of constant conductivity of the layer, the values of $K_{0} C k_{n}^{m}$ can be estimated from the observed magnetic potential [15]. Since the velocity potential $\psi_{n}^{n}$ contributes to the current function $R_{n+1}^{n}$, a solution in region II, using the result obtained by $M$. Hasegawa and $M$. Ota [3] (Table 1), becomes 


$$
\begin{aligned}
R_{I I}=\sum_{n} f R_{n+1}^{n} & \sum_{\mu}(-1)^{\mu}\left[S_{n}^{\mu}+\frac{C_{I I}^{\mu}}{c_{m}^{\mu}} p^{\mu}+\frac{C_{I I I}^{\mu}}{c_{m}^{\mu}} q^{\mu}\right] \\
\times & {\left[\frac{1}{2}\left(A_{\mu_{n}}-B_{\mu_{n}}\right) \sin \left(n T+\mu \Lambda+e_{n+1}^{n}\right)\right.} \\
+ & \left.\frac{1}{2}\left(A_{\mu_{n}}+B_{\mu_{n}}\right) \sin \left(n T-\mu \Lambda+e_{n+1}^{n}\right)\right],
\end{aligned}
$$

where

$$
R_{n+1}^{n}=-\frac{1}{4 \pi} \frac{2 n+3}{n+2}\left(\frac{a}{r_{0}}\right)^{n+1} r_{0} E_{n+1}^{n}
$$

Fig. 2 shows the lines of eastward maximum current density an $11 \mathrm{~h} 16 \mathrm{~m}$ local time in the cases of $f=1, f=5$, and $f=100$, and Fig. 3 the variation of current density along its line in the case of $f=5$. Fig. 5 shows a departure of the line of maximum current density from the geographic equator on the meridian of $\Lambda=0$. From these Figures we have found that;

(i) a line of the maximum current density for $\Theta_{1}=80^{\circ}$ (as an example) and $f=5^{\prime}$ is in good agreement with the above-mentioned average equator for $S_{7}$,

(ii) the value for current density along the line of its maximum is large at the places where the geomagnetic equator is situated far from the geographic equator, but the range of variation with longitude is merely 1.4 per cent,

(iii) unless the highly conductive zone II is not very narrow, the position of maximum density may be shifted fairly well from the middle of the highly conductive zone.

\section{Conclusion}

Above considerations seem to suggest the fairly deep-rooted effect of the dynamo-action due to the discrepancy between the above two axes upon the $S_{7}$-field on one hand, and the high conductivity in the equatorial zone (several times higher than in the middle latitude) on the other.

\section{Acknowledgements}

In conclusion, the author wishes to express his hearty thanks to Prof. M. Hasegawa for his direction throughout this study, and to Mr. M. Hirono for his valuable advice.

(Read Oct. 21, 1951)

\section{References}

[1] Maeda, H., J. Geomag. Geoelec., 5. 39 (1953).

[2] Johnston, H,F. \& McNish, A.G., C.R. Cong. Internat. Electricité, Paris, 12, 41 (1932).

[ 3 ] Hasegawa, M. \& Ota, M., Trans. Oslo Meeting 1948, I.A.T.M.E. Bull. No. 13, 426 (1950), 
[4] Price, A.T. \& Wilkins, G.A., J. Geophys. Res., 56, 259 (1951).

[5] Egedal, J., Terr. Mag., 52, 449 (1947); Nature, 161, 443 (1948); Trans. Brussels Meeting 1951, I.A.T.M.E. (to be published).

[6] Gulatee, B.L., Surv. Ind. Tech. Rep., Pt. 3 (1949-50).

[7] Pramanik, S.K. \& Narayanan, S.Y., Ind. Jour. Meteorol. Geophys., 3, 49 (1952).

[8] Chapman, S., Proc. Phys. Soc. B. 64, 833 (1951).

[9] McNish, A.G., Trans. Edinburgh Meeting 1936, I.A.T.M.E. Bull. No. 10, 271 (1937).

[10] Martyn, D.F., Nature. 162, 142 (1948); Trans. Oslo Meeting 1948, I.A.T.M.E. Bull. No. 13, 431 (1950).

[11] Hirono, M., J. Geomag. Geoelec., 2, 1 \& 113 (1950); Ibid. 4.7 (1952).

[12] Maeda, K., J. Geomag. Geoelec., 4, 63 (1952).

[13] Cowling, T.G., Report to The 3rd (Camberra) Meeting 1952, Mixed Commission on the Ionosphere, I.C.S.U. (to be published).

[14] Martyn D.F. \& Baker, W.G., Nature, 170, 1090 (1952); Proc. Roy. Soc. A (to be published).

[15] Nagata, T. \& Sugiura, M., Geophys. Notes, Vol. 1, No. 36 (1948).

The Meeting of the Society of Terrestrial Magnetism and Electricity :

The 13th General Meeting was held at the Geographic Survey Institute on May 15-17, 1953.

58 Reports were read, about 100 Members assembled,

The Tanakadate-Prize was awarded for the following excellent workers:

The 13th, Mr. S. Uyeda and Mr. S. Akimoto, at the 13th General Meeting :

Self-Reversal of Thermo-Remanent Magnetism of Igneous Rocks. 